https://doi.org/10.15407/ujpe63.2.102

KH.P. GNATENKO, V.M. TKACHUK

Department for Theoretical Physics, Ivan Franko National University of Lviv

(12, Drahomanov Str., Lviv 79005, Ukraine; e-mail: khrystyna.gnatenko@gmail.com)

\title{
LENGTH IN A NONCOMMUTATIVE PHASE SPACE
}

We study restrictions on the length in a noncommutative phase space caused by noncommutativity. The uncertainty relations for coordinates and momenta are considered, and the lower bound of the length is found. We also consider the eigenvalue problem for the squared length operator and find the expression for the minimal length in the noncommutative phase space.

Keywords: noncommutative phase space, minimal length, uncertainty relations.

\section{Introduction}

Due to the development of String Theory and Quantum Gravity [1, 2]), the studies of physical systems in the framework of noncommutative quantum and classical mechanics attract a great interest [3-15]. It is worth noting that the idea of noncommutativity is quite old, has been proposed by W. Heisenberg, and formalized by H. Snyder in work [16].

Much attention has been devoted to the influence of noncommutativity on the properties of physical systems, among them are hydrogen atom [18-20, 34], harmonic oscillator [21-27,34], particle in a gravitational quantum well $[28,29]$, composite systems [30], etc.

In the general case, the noncommutative phase space can be realized with the following commutation relations for coordinates and momenta:

$\left[X_{i}, X_{j}\right]=i \hbar \theta_{i j}$

$\left[X_{i}, P_{j}\right]=i \hbar \delta_{i j}+i \hbar \gamma_{i j}$

$\left[P_{i}, P_{j}\right]=i \hbar \eta_{i j}$.

Here, $\theta_{i j}$ and $\eta_{i j}$ are elements of the constant antisymmetric matrices called the parameters of noncommutativity of the coordinates and momenta, respectively. The parameters $\gamma_{i j}$ are elements of a constant matrix.

(C) KH.P. GNATENKO, V.M. TKACHUK, 2018

102
Note that the coordinates $X_{i}$ and the momenta $P_{i}$, which satisfy relations (1) and (3), can be represented as

$X_{i}=x_{i}-\frac{1}{2} \sum_{j} \theta_{i j} p_{j}$,

$P_{i}=p_{i}+\frac{1}{2} \sum_{j} \eta_{i j} x_{j}$,

with $x_{i}, p_{i}$ being coordinates and momenta, which satisfy the ordinary commutation relations

$\left[x_{i}, x_{j}\right]=0$,

$\left[x_{i}, p_{j}\right]=i \hbar \delta_{i j}$

$\left[p_{i}, p_{j}\right]=0$.

Using (4) and (5), we obtain

$\left[X_{i}, P_{j}\right]=i \hbar \delta_{i j}+i \hbar \sum_{k} \frac{\theta_{i k} \eta_{j k}}{4}$

So, we consider $\gamma_{i j}$ to be defined as

$\gamma_{i j}=\sum_{k} \frac{\theta_{i k} \eta_{j k}}{4}$.

In view of the commutation relations (1)-(3), one can write the following uncertainty relations:

$\left\langle\Delta X_{i}^{2}\right\rangle\left\langle\Delta X_{j}^{2}\right\rangle \geq \frac{\hbar^{2} \theta_{i j}^{2}}{4}$,

ISSN 2071-0194. Ukr. J. Phys. 2018. Vol. 63, No. 2 
$\left\langle\Delta P_{i}^{2}\right\rangle\left\langle\Delta P_{j}^{2}\right\rangle \geq \frac{\hbar^{2} \eta_{i j}^{2}}{4}$,

$\left\langle\Delta X_{i}^{2}\right\rangle\left\langle\Delta P_{j}^{2}\right\rangle \geq \frac{\hbar^{2}\left(\delta_{i j}+2 \gamma_{i j} \delta_{i j}+\gamma_{i j}^{2}\right)}{4}$.

Note that there are no summations over indices $i$ and $j$ in (13). So, in the noncommutative phase space, there are additional limits to the precision, with which coordinates and momenta can be known. This leads to additional bounds of the physical values in a space with noncommutativity of coordinates and noncommutativity of momenta.

In the present paper, we consider a length in the noncommutative phase space. We study lower bounds of the length caused by the noncommutativity. For this purpose, the uncertainty relations are considered, and the eigenvalue problem for the squared length operator is examined. We find an expression for the minimal length in the noncommutative phase space.

The article is organized as follows. In Section 2, the squared length operator defined in the coordinate space is studied. We consider restrictions on the length caused by the noncommutativity. In Section 2, we examine the length defined in the momentum space and find lower bounds on its value. Section 4 is devoted to studies of a length defined in the phase space. The eigenvalue problem for the squared length operator is examined, and the minimal length in the phase space is found. Conclusions are presented in Section 5.

\section{Length in the Coordinate Space}

Let us first examine lower bounds on the length, which is defined in the two-dimensional coordinate space. For this purpose, we consider the squared length operator

$R_{12}^{2}=X_{1}^{2}+X_{2}^{2}$,

and write its eigenvalues. The coordinates $X_{1}$ and $X_{2}$ satisfy the commutation relation

$\left[X_{1}, X_{2}\right]=i \hbar \theta_{12}$

Note that $X_{1}, X_{2}$ can be represented as

$X_{1}=x_{1}-\frac{1}{2} \theta_{12} p_{2}$,

$X_{2}=x_{2}+\frac{1}{2} \theta_{12} p_{1}$.

ISSN 2071-0194. Ukr. J. Phys. 2018. Vol. 63, No. 2
Here, the coordinates $x_{i}$ and the momenta $p_{i}$ satisfy the ordinary commutation relations

$\left[x_{1}, x_{2}\right]=0$,

$\left[p_{1}, p_{2}\right]=0$,

$\left[x_{1}, p_{1}\right]=\left[x_{2}, p_{2}\right]=i \hbar$.

So, we can rewrite the operator $R_{12}^{2}$ in the following form:

$R_{12}^{2}=x_{1}^{2}+x_{2}^{2}+\frac{\theta_{12}^{2}}{4}\left(p_{1}^{2}+p_{2}^{2}\right)-\theta_{12}\left(x_{1} p_{2}-x_{2} p_{1}\right)$.

Note that $R_{12}^{2}$ can be factorized. Considering the operators (see, e.g., $[26,32]$ )

$b_{1}=\frac{1}{2}\left(-i \xi_{1}-i \frac{d}{d \xi_{1}}+\xi_{2}+\frac{d}{d \xi_{2}}\right)$,

$b_{1}^{+}=\frac{1}{2}\left(i \xi_{1}-i \frac{d}{d \xi_{1}}+\xi_{2}-\frac{d}{d \xi_{2}}\right)$,

with dimensionless coordinates

$\xi_{1}=\frac{\sqrt{2}}{\sqrt{\hbar\left|\theta_{12}\right|}} x_{1}$,

$\xi_{2}=\frac{\sqrt{2}}{\sqrt{\hbar\left|\theta_{12}\right|}} x_{2}$,

we can write

$R_{12}^{2}=2 \hbar\left|\theta_{12}\right|\left(b_{1}^{+} b_{1}+\frac{1}{2}\right)$.

The operators $b_{1}, b_{1}^{+}$satisfy the commutation relation

$\left[b_{1}, b_{1}^{+}\right]=1$.

Therefore, the eigenvalues of $R_{12}^{2}$ read [31]

$r_{n_{12}}^{2}=2 \hbar\left|\theta_{12}\right|\left(n_{12}+\frac{1}{2}\right)$,

where $n_{12}$ is a quantum number: $n_{12}=0,1,2,3, \ldots$. So, taking (28) into account, we can write the inequalities

$\left\langle\Delta R_{12}^{2}\right\rangle \geq \hbar\left|\theta_{12}\right|$,

$\Delta R_{12} \geq \sqrt{\hbar\left|\theta_{12}\right|}$,

where $\left\langle X_{1}\right\rangle=\left\langle X_{2}\right\rangle=0$,

$\left\langle\Delta R_{12}^{2}\right\rangle=\left\langle\Delta X_{1}^{2}\right\rangle+\left\langle\Delta X_{2}^{2}\right\rangle$, 
$\Delta R_{12}=\sqrt{\left\langle\Delta R_{12}^{2}\right\rangle}$.

Similarly, for the operators

$R_{23}^{2}=X_{2}^{2}+X_{3}^{2}$,

$R_{31}^{2}=X_{3}^{2}+X_{1}^{2}$,

where the coordinates $X_{i}, X_{j}$ satisfy the commutation relations (1), we can write the eigenvalues

$r_{n_{23}}^{2}=2 \hbar\left|\theta_{23}\right|\left(n_{23}+\frac{1}{2}\right)$,

$r_{n_{31}}^{2}=2 \hbar\left|\theta_{31}\right|\left(n_{31}+\frac{1}{2}\right)$,

with $n_{23}$ and $n_{31}$ being quantum numbers, and obtain the inequalities

$\left\langle\Delta R_{23}^{2}\right\rangle \geq \hbar\left|\theta_{23}\right|, \quad \Delta R_{23} \geq \sqrt{\hbar\left|\theta_{23}\right|}$,
$\left\langle\Delta R_{31}^{2}\right\rangle \geq \hbar\left|\theta_{31}\right|, \quad \Delta R_{31} \geq \sqrt{\hbar\left|\theta_{31}\right|}$.

Here, $\left\langle X_{1}\right\rangle=\left\langle X_{2}\right\rangle=\left\langle X_{3}\right\rangle=0$,

$\left\langle\Delta R_{i j}^{2}\right\rangle=\left\langle\Delta X_{i}^{2}\right\rangle+\left\langle\Delta X_{j}^{2}\right\rangle$,

$\Delta R_{i j}=\sqrt{\left\langle\Delta R_{i j}^{2}\right\rangle}$.

With regard for (29), (37), and (38), we can conclude that there are restrictions on the length caused by the noncommutativity. It is worth noting that, in the general case, $\left|\theta_{12}\right| \neq\left|\theta_{23}\right| \neq\left|\theta_{31}\right|$. So, the restriction on the length is anisotropic.

Let us study the squared length operator defined in a three-dimensional coordinate space

$\mathbf{R}^{2}=\sum_{i} X_{i}^{2}$.

Note that the coordinates $X_{i}$ do not commute (1). In view of (41), we can writes

$\left\langle\Delta \mathbf{R}^{2}\right\rangle=\left\langle\Delta X_{1}^{2}\right\rangle+\left\langle\Delta X_{2}^{2}\right\rangle+\left\langle\Delta X_{3}^{2}\right\rangle$.

To find restrictions on the value $\left\langle\Delta \mathbf{R}^{2}\right\rangle$ and, as a result, to obtain restrictions on the value of length $\Delta R=\sqrt{\left\langle\Delta \mathbf{R}^{2}\right\rangle}$, let us write eigenvalues of $\mathbf{R}^{2}$. Using the representation for the noncommutative coordinates

$X_{i}=x_{i}-\frac{1}{2} \sum_{j} \theta_{i j} p_{j}$ with the coordinates $x_{i}$ and $p_{i}$ satisfying the ordinary commutation relations $(6)-(8)$, we can write

$\mathbf{R}^{2}=\mathbf{x}^{2}+\frac{1}{4}[\boldsymbol{\theta} \times \mathbf{p}]^{2}-(\boldsymbol{\theta} \mathbf{L})=$

$=\mathbf{x}^{2}+\frac{1}{4} \theta^{2} p^{2}-\frac{1}{4}(\boldsymbol{\theta} \mathbf{p})^{2}-(\boldsymbol{\theta} \mathbf{L})$.

Here, $\mathbf{x}^{2}=\sum_{i} x_{i}^{2}$, and the components of the vector $\boldsymbol{\theta}$ are defined as

$\theta_{k}=\frac{1}{2} \sum_{i, j} \varepsilon_{i j k} \theta_{i j}$.

It is worth noting that two first terms in (44) are invariant under rotation. So, it is convenient to choose a frame of reference so that the directions of the $x_{3}$ axis and the vector $\boldsymbol{\theta}$ coincide. In this case, we can write

$\mathbf{R}^{2}=\mathbf{x}^{2}+\frac{1}{4}[\boldsymbol{\theta} \times \mathbf{p}]^{2}-\theta\left(x_{1} p_{2}-x_{2} p_{1}\right)=$
$=x_{1}^{2}+x_{2}^{2}+x_{3}^{2}+\frac{1}{4} \theta^{2} p_{1}^{2}+\frac{1}{4} \theta^{2} p_{2}^{2}-\theta\left(x_{1} p_{2}-x_{2} p_{1}\right)$

with

$\theta=|\boldsymbol{\theta}|=\sqrt{\theta_{12}^{2}+\theta_{23}^{2}+\theta_{31}^{2}}$.

In (46), we use the same notations for the coordinates $x_{i}$ in the chosen frame of reference. Note that $x_{3}^{2}$ commutes with $\mathbf{R}^{2}$. So, the eigenvalues of $\mathbf{R}^{2}$ read

$R^{2}=2 \hbar \theta\left(n+\frac{1}{2}\right)+r_{3}^{2}$.

Here, $r_{3}^{2}$ are eigenvalues of the operator $x_{3}^{2}$, and $n$ are the quantum number: $n=0,1,2 \ldots$. In view of (48), we can writes

$\left\langle\Delta \mathbf{R}^{2}\right\rangle \geq \hbar \theta$.

So, we obtain the inequality

$\Delta R \geq \sqrt{\hbar \theta}$,

$\Delta R=\sqrt{\left\langle\Delta \mathbf{R}^{2}\right\rangle}$,

which imposes a restriction on the length in the threedimensional noncommutative space.

Note that, adding inequalities (29), (37), and (38), we get

$\left\langle\Delta \mathbf{R}^{2}\right\rangle=\left\langle\Delta X_{1}^{2}\right\rangle+\left\langle\Delta X_{2}^{2}\right\rangle+\left\langle\Delta X_{3}^{2}\right\rangle \geq$

$\geq \frac{\hbar}{2}\left(\left|\theta_{12}\right|+\left|\theta_{23}\right|+\left|\theta_{31}\right|\right)$,

ISSN 2071-0194. Ukr. J. Phys. 2018. Vol. 63, No. 2 
Relation (52) yields

$\Delta R \geq \sqrt{\frac{\hbar}{2}\left(\left|\theta_{12}\right|+\left|\theta_{23}\right|+\left|\theta_{31}\right|\right)}$.

On the other hand, the restriction on the value $\Delta R$ can be found, by using the uncertainty relations (11) [33]. Taking (11) and (42) into account and making algebraic transformations, we can write

$$
\begin{aligned}
& \left\langle\Delta \mathbf{R}^{2}\right\rangle^{2} \geq 2\left\langle\Delta X_{1}^{2}\right\rangle\left\langle\Delta X_{2}^{2}\right\rangle+2\left\langle\Delta X_{2}^{2}\right\rangle\left\langle\Delta X_{3}^{2}\right\rangle+ \\
& +2\left\langle\Delta X_{3}^{2}\right\rangle\left\langle\Delta X_{1}^{2}\right\rangle \geq \frac{\hbar^{2}}{2}\left(\theta_{12}^{2}+\theta_{23}^{2}+\theta_{31}^{2}\right),
\end{aligned}
$$

which leads to

$\Delta R \geq\left(\frac{\hbar^{2}}{2}\left(\theta_{12}^{2}+\theta_{23}^{2}+\theta_{31}^{2}\right)\right)^{1 / 4}$.

Let us compare the obtained results (50), (53), and (55). It is clear that the lower bound $\hbar^{1 / 2}\left(\theta_{12}^{2}+\right.$ $\left.+\theta_{23}^{2}+\theta_{31}^{2}\right)^{1 / 4}$ presented by (50) is stronger than $\left(\frac{\hbar^{2}}{2}\left(\theta_{12}^{2}+\theta_{23}^{2}+\theta_{31}^{2}\right)\right)^{1 / 4}$ which is given in (55). To compare (50) and (53), we consider

$\hbar^{2}\left(\theta_{12}^{2}+\theta_{23}^{2}+\theta_{31}^{2}\right)-\frac{\hbar^{2}}{4}\left(\left|\theta_{12}\right|+\left|\theta_{23}\right|+\left|\theta_{31}\right|\right)^{2}=$

$=\frac{\hbar^{2}}{4}\left(\left|\theta_{12}\right|-\left|\theta_{23}\right|-\left|\theta_{31}\right|\right)^{2}+\frac{\hbar^{2}}{4}\left(\left|\theta_{23}\right|-\left|\theta_{12}\right|-\right.$

$\left.-\left|\theta_{31}\right|\right)^{2}+\frac{\hbar^{2}}{4}\left(\left|\theta_{31}\right|-\left|\theta_{23}\right|-\left|\theta_{12}\right|\right)^{2} \geq 0$.

Therefore,

$\hbar^{1 / 2}\left(\theta_{12}^{2}+\theta_{23}^{2}+\theta_{31}^{2}\right)^{1 / 4} \geq\left(\frac{\hbar}{2}\right)^{1 / 2}\left(\left|\theta_{12}\right|+\left|\theta_{23}\right|+\left|\theta_{31}\right|\right)^{1 / 2}$.

So, inequality (50) imposes a stronger restriction on the length in the noncommutative space than (53) and (55). The minimal length in the noncommutative space reads

$\Delta R^{\min }=\hbar^{1 / 2}\left(\theta_{12}^{2}+\theta_{23}^{2}+\theta_{31}^{2}\right)^{1 / 4}$.

\section{Length in the Momentum Space}

Let us study the squared length operator defined in the momentum space. Let us first consider the twodimensional case. We have

$P_{12}^{2}=P_{1}^{2}+P_{2}^{2}$.
Here, the momenta $P_{1}, P_{2}$ do not commute

$\left[P_{1}, P_{2}\right]=i \hbar \eta_{12}$.

Let us use the representation for noncommutative momenta

$P_{1}=p_{1}+\frac{1}{2} \eta_{12} x_{2}$

$P_{2}=p_{2}-\frac{1}{2} \eta_{12} x_{1}$.

The commutation relations for the operators $x_{i}$ and $p_{i}$ are as follows $\left[x_{1}, x_{2}\right]=0,\left[p_{1}, p_{2}\right]=0,\left[x_{1}, p_{1}\right]=$ $=\left[x_{2}, p_{2}\right]=i \hbar$. Using the above-given representation, we have

$P_{12}^{2}=p_{1}^{2}+p_{2}^{2}+\frac{\eta_{12}^{2}}{4}\left(x_{1}^{2}+x_{2}^{2}\right)-\eta_{12}\left(x_{1} p_{2}-x_{2} p_{1}\right)$,

Like the previous section in the case of the operator $R_{12}^{2}$, we introduce the dimensionless coordinates $\xi_{1}=\sqrt{\eta_{12}} x_{1} / \sqrt{2 \hbar}, \xi_{2}=\sqrt{\eta_{12}} x_{2} / \sqrt{2 \hbar}$. Considering the operators (22) and (23), we can write

$P_{12}^{2}=2 \hbar\left|\eta_{12}\right|\left(b_{1}^{+} b_{1}+\frac{1}{2}\right)$.

The eigenvalues of $P_{12}^{2}$ read [34]

$p_{m_{12}}^{2}=2 \hbar\left|\eta_{12}\right|\left(m_{12}+\frac{1}{2}\right)$

with $m_{12}$ being a quantum number: $m_{12}=0,1,2$, $3, \ldots$ So, the following inequality can be written:

$\left\langle\Delta P_{1}^{2}\right\rangle+\left\langle\Delta P_{2}^{2}\right\rangle \geq \hbar\left|\eta_{12}\right|$

$\Delta P_{12} \geq \sqrt{\hbar\left|\eta_{12}\right|}$

where $\left\langle P_{1}\right\rangle=\left\langle P_{2}\right\rangle=0$ and $\Delta P_{12}=\sqrt{\left\langle\Delta P_{12}^{2}\right\rangle}$. Analogously, the eigenvalues of the operators

$P_{23}^{2}=P_{2}^{2}+P_{3}^{2}$,

$P_{31}^{2}=P_{3}^{2}+P_{1}^{2}$

read

$p_{m_{23}}^{2}=2 \hbar\left|\eta_{23}\right|\left(m_{23}+\frac{1}{2}\right)$

$p_{m_{31}}^{2}=2 \hbar\left|\eta_{31}\right|\left(m_{31}+\frac{1}{2}\right)$

where $m_{23}$ and $m_{31}$ are quantum numbers: $m_{23}=0$, $1,2,3, \ldots, m_{31}=0,1,2,3, \ldots$. So, we can write

$\Delta P_{23} \geq \sqrt{\hbar\left|\eta_{23}\right|}$ 
$\Delta P_{31} \geq \sqrt{\hbar\left|\eta_{31}\right|}$

with $\Delta P_{i j}=\sqrt{\left\langle\Delta P_{i j}^{2}\right\rangle}$.

In the general case, $\left|\eta_{12}\right| \neq\left|\eta_{23}\right| \neq\left|\eta_{31}\right|$. So, there is the anisotropy of restrictions on the length defined in the momentum space. This anisotropy is caused by the anisotropy of parameters of the momentum noncommutativity (3).

On the basis of inequalities (67), (72), and (73), we get

$$
\begin{aligned}
& \left\langle\Delta \mathbf{P}^{2}\right\rangle \geq \frac{\hbar}{2}\left(\left|\eta_{12}\right|+\left|\eta_{23}\right|+\left|\eta_{31}\right|\right), \\
& \Delta P \geq \sqrt{\frac{\hbar}{2}\left(\left|\eta_{12}\right|+\left|\eta_{23}\right|+\left|\eta_{31}\right|\right)} .
\end{aligned}
$$

In the three-dimensional case, we have

$\mathbf{P}^{2}=P_{1}^{2}+P_{2}^{2}+P_{3}^{2}$.

To find the eigenvalues of operator (76), let us use the representation for noncommutative momenta

$P_{i}=p_{i}+\frac{1}{2} \sum_{j} \eta_{i j} x_{j}$

with the coordinates $x_{i}$ and $p_{i}$ satisfying the ordinary commutation relations (6)-(8). So, we can rewrite $\mathbf{P}^{2}$ as

$\mathbf{P}^{2}=\mathbf{p}^{2}+\frac{1}{4} \eta^{2} p^{2}-\frac{1}{4}(\boldsymbol{\eta} \mathbf{x})^{2}-(\boldsymbol{\eta} \mathbf{L})$,

where the components of the vector $\boldsymbol{\eta}$ are defined as

$\eta_{k}=\frac{1}{2} \sum_{i, j} \varepsilon_{i j k} \eta_{i j}$

$\eta=|\boldsymbol{\eta}|=\sqrt{\eta_{12}^{2}+\eta_{23}^{2}+\eta_{31}^{2}}$

Note that two first terms in (78) are rotationally invariant. Therefore, we can write

$\mathbf{P}^{2}=\mathbf{p}^{2}+\frac{1}{4}[\boldsymbol{\eta} \times \mathbf{x}]^{2}-\eta\left(x_{1} p_{2}-x_{2} p_{1}\right)=$

$=p_{1}^{2}+p_{2}^{2}+p_{3}^{2}+\frac{1}{4} \eta^{2} x_{1}^{2}+\frac{1}{4} \eta^{2} x_{2}^{2}-\eta\left(x_{1} p_{2}-x_{2} p_{1}\right)$.

Here, we have chosen a frame of reference with the coincidence of the $x_{3}$-axis direction and the direction of the vector $\boldsymbol{\eta}$. It is worth noting that $\left[p_{3}^{2}, \mathbf{P}^{2}\right]=0$. So, the eigenvalues of $\mathbf{P}^{2}$ read

$P^{2}=2 \hbar \eta\left(m+\frac{1}{2}\right)+\hbar^{2} k^{2}$, where $m$ is the quantum number: $m=0,1,2, \ldots$, and $\hbar^{2} k^{2}$ are eigenvalues of the operator $p_{3}^{2}$. Using expression (82) for the eigenvalues, we can write

$\left\langle\Delta \mathbf{P}^{2}\right\rangle \geq \hbar \eta$.

Here, we consider that $\left\langle P_{i}\right\rangle=0$.

So, the restriction on the length defined in the noncommutative momentum space is given by the inequality

$\Delta P \geq \sqrt{\hbar \eta}$

Note that

$\hbar^{1 / 2}\left(\eta_{12}^{2}+\eta_{23}^{2}+\eta_{31}^{2}\right)^{1 / 4} \geq \hbar^{1 / 2}\left(\frac{1}{2}\left(\eta_{12}+\eta_{23}+\eta_{31}\right)\right)^{1 / 2}$.

So, the minimal length in the noncommutative momentum space reads

$\Delta P^{\min }=\hbar^{1 / 2}\left(\eta_{12}^{2}+\eta_{23}^{2}+\eta_{31}^{2}\right)^{1 / 4}$.

\section{Length in the Phase Space}

In the general case, the squared length operator can be defined in noncommutative phase space as

$\mathbf{Q}^{2}=\alpha^{2} \sum_{i} P_{i}^{2}+\beta^{2} \sum_{i} X_{i}^{2}$,

where

$\mathbf{Q}=\alpha \mathbf{P}+\beta \mathbf{X}$

and $\alpha$ and $\beta$ are constants. By the dimensional cause, the constant $\beta$ is dimensionless and $\alpha$ has dimension $s / k g$. Coordinates $X_{i}$ and momenta $P_{i}$ satisfy the noncommutative algebra (1)-(3). Note that operator (87) can be also considered as the Hamiltonian of a harmonic oscillator in the noncommutative phase space.

First, let us consider the two-dimensional case. Therefore, we have

$Q_{12}^{2}=\alpha^{2}\left(P_{1}^{2}+P_{2}^{2}\right)+\beta^{2}\left(X_{1}^{2}+X_{2}^{2}\right)$.

Operator (89) corresponds to the Hamiltonian of a two-dimensional harmonic oscillator in the noncommutative phase space. Using representation (16), (17), (61), (62), we can write

$Q_{12}^{2}=\left(\alpha^{2}+\frac{\theta_{12}^{2} \beta^{2}}{4}\right)\left(p_{1}^{2}+p_{2}^{2}\right)+\left(\beta^{2}+\frac{\eta_{12}^{2} \alpha^{2}}{4}\right) \times$
$\times\left(x_{1}^{2}+x_{2}^{2}\right)-\left(\eta_{12} \alpha^{2}+\theta_{12} \beta^{2}\right)\left(x_{1} p_{2}-x_{2} p_{1}\right)$,

ISSN 2071-0194. Ukr. J. Phys. 2018. Vol. 63, No. 2 
Introducing the set of operators (see, e.g., [26, 32])

$b_{1}=\frac{1}{2}\left(-i \xi_{1}-i \frac{d}{d \xi_{1}}+\xi_{2}+\frac{d}{d \xi_{2}}\right)$,

$b_{1}^{+}=\frac{1}{2}\left(i \xi_{1}-i \frac{d}{d \xi_{1}}+\xi_{2}-\frac{d}{d \xi_{2}}\right)$,

$b_{2}=\frac{1}{2}\left(-i \xi_{1}-i \frac{d}{d \xi_{1}}-\xi_{2}-\frac{d}{d \xi_{2}}\right)$,

$b_{2}^{+}=\frac{1}{2}\left(i \xi_{1}-i \frac{d}{d \xi_{1}}-\xi_{2}+\frac{d}{d \xi_{2}}\right)$,

where $\xi_{1}=l_{0} x_{1}, \xi_{2}=l_{0} x_{2}$ with

$l_{0}=\hbar^{\frac{1}{2}}\left(\frac{4 \alpha^{2}+\theta_{12}^{2} \beta^{2}}{4 \beta^{2}+\eta_{12}^{2} \alpha^{2}}\right)^{1 / 4}$,

the operator $Q_{12}^{2}$ can be written as

$$
\begin{aligned}
& Q_{12}^{2}=\hbar\left(\sqrt{\left(2 \alpha^{2}+\frac{\theta_{12}^{2} \beta^{2}}{2}\right)\left(2 \beta^{2}+\frac{\eta_{12}^{2} \alpha^{2}}{2}\right)}+\right. \\
& \left.+\eta_{12} \alpha^{2}+\theta_{12} \beta^{2}\right) b_{1}^{+} b_{1}+ \\
& +\hbar\left(\sqrt{\left(2 \alpha^{2}+\frac{\theta_{12}^{2} \beta^{2}}{2}\right)\left(2 \beta^{2}+\frac{\eta_{12}^{2} \alpha^{2}}{2}\right)}-\right. \\
& \left.-\eta_{12} \alpha^{2}-\theta_{12} \beta^{2}\right) b_{2}^{+} b_{2}+ \\
& +\hbar \sqrt{\left(2 \alpha^{2}+\frac{\theta_{12}^{2} \beta^{2}}{2}\right)\left(2 \beta^{2}+\frac{\eta_{12}^{2} \alpha^{2}}{2}\right)} .
\end{aligned}
$$

Note that the following commutation relations are satisfied:

$$
\begin{aligned}
& {\left[b_{1}, b_{1}^{+}\right]=\left[b_{2}, b_{2}^{+}\right]=1,} \\
& {\left[b_{1}, b_{2}^{+}\right]=\left[b_{2}, b_{1}^{+}\right]=\left[b_{1}, b_{2}\right]=\left[b_{2}^{+}, b_{1}^{+}\right]=0 .}
\end{aligned}
$$

So, with regard for (96), (97), and (98), the eigenvalues of $Q_{12}^{2}$ read [26]

$$
\begin{aligned}
& Q_{12, n_{1} n_{2}}^{2}=\hbar\left(\sqrt{\left(2 \alpha^{2}+\frac{\theta_{12}^{2} \beta^{2}}{2}\right)\left(2 \beta^{2}+\frac{\eta_{12}^{2} \alpha^{2}}{2}\right)}+\right. \\
& \left.+\eta_{12} \alpha^{2}+\theta_{12} \beta^{2}\right)\left(n_{1}+1\right)+ \\
& +\hbar\left(\sqrt{\left(2 \alpha^{2}+\frac{\theta_{12}^{2} \beta^{2}}{2}\right)\left(2 \beta^{2}+\frac{\eta_{12}^{2} \alpha^{2}}{2}\right)}-\right. \\
& \left.-\eta_{12} \alpha^{2}-\theta_{12} \beta^{2}\right) n_{2} .
\end{aligned}
$$

ISSN 2071-0194. Ukr. J. Phys. 2018. Vol. 63, No. 2
Using (99), we can write the inequality

$\Delta Q_{i j} \geq\left(\hbar\left(2 \alpha+\frac{\theta_{i j}^{2} \beta}{2}\right)^{1 / 2}\left(2 \beta+\frac{\eta_{i j}^{2} \alpha}{2}\right)^{1 / 2}+\right.$

$\left.+\hbar \eta_{i j} \alpha+\hbar \theta_{i j} \beta\right)^{1 / 2}$.

Here,

$\Delta Q_{i j}=\sqrt{\left\langle\Delta Q_{i j}^{2}\right\rangle}=$

$=\sqrt{\alpha^{2}\left\langle\Delta P_{i}^{2}\right\rangle+\alpha^{2}\left\langle\Delta P_{j}^{2}\right\rangle+\beta^{2}\left\langle\Delta X_{i}^{2}\right\rangle+\beta^{2}\left\langle\Delta X_{j}^{2}\right\rangle}$,

$\left\langle X_{i}\right\rangle=0,\left\langle P_{i}\right\rangle=0, i, j=(1,2,3)$. So, the minimal length reads

$\Delta Q_{i j}^{\min }=\left(\hbar\left(2 \alpha^{2}+\frac{\theta_{i j}^{2} \beta^{2}}{2}\right)^{1 / 2}\left(2 \beta^{2}+\frac{\eta_{i j}^{2} \alpha^{2}}{2}\right)^{1 / 2}+\right.$

$\left.+\hbar \eta_{i j} \alpha^{2}+\hbar \theta_{i j} \beta^{2}\right)^{1 / 2}$.

Note that, in the general case, $\Delta Q_{12}^{\min } \neq \Delta Q_{23}^{\min } \neq$ $\neq \Delta Q_{31}^{\min }$ because of $\left|\eta_{12}\right| \neq\left|\eta_{23}\right| \neq\left|\eta_{31}\right|$.

In the three-dimensional phase space, we have

$\mathbf{Q}^{2}=\alpha^{2}\left(P_{1}^{2}+P_{2}^{2}+P_{3}^{2}\right)+\beta^{2}\left(X_{1}^{2}+X_{2}^{2}+X_{3}^{2}\right)$.

Using the representation for the noncommutative coordinates and noncommutative momenta (4)-(5), we obtain

$\mathbf{Q}^{2}=\left(\alpha^{2}+\frac{\beta^{2}}{4} \theta^{2}\right) p^{2}+\left(\beta^{2}+\frac{\alpha^{2}}{4} \eta^{2}\right) x^{2}-$

$-\frac{\alpha^{2}}{4}(\boldsymbol{\eta} \mathbf{x})^{2}-\frac{\beta^{2}}{4}(\boldsymbol{\theta} \mathbf{p})^{2}-\alpha^{2}(\boldsymbol{\eta} \mathbf{L})-\beta^{2}(\boldsymbol{\theta} \mathbf{L})$.

It is convenient to choose a frame of reference, by considering the coincidence of the directions of the $x_{3}$-axis and the vector $\alpha^{2} \boldsymbol{\eta}+\beta^{2} \boldsymbol{\theta}$. We also consider the vectors $\boldsymbol{\theta}$ and $\boldsymbol{\eta}$ to have the same direction

$\boldsymbol{\theta} \| \boldsymbol{\eta}$

In this case, preserving notations for coordinates and momenta, we have

$$
\begin{aligned}
& \mathbf{Q}^{2}=\left(\alpha^{2}+\frac{\beta^{2}}{4} \theta^{2}\right)\left(p_{1}^{2}+p_{2}^{2}\right)+\left(\beta^{2}+\frac{\alpha^{2}}{4} \eta^{2}\right) \times \\
& \times\left(x_{1}^{2}+x_{2}^{2}\right)+\alpha^{2} p_{3}^{2}+\beta^{2} x_{3}^{2}-\left(\alpha^{2} \eta+\beta^{2} \theta\right) \times \\
& \times\left(x_{1} p_{2}-x_{2} p_{1}\right) .
\end{aligned}
$$


Note that the operator $\alpha^{2} p_{3}^{2}+\beta^{2} x_{3}^{2}$, which corresponds to the Hamiltonian of a harmonic oscillator in the ordinary space, commutes with $\mathbf{Q}^{2}$. Note also that other terms in $\mathbf{Q}^{2}$,

$\left(\alpha^{2}+\frac{\beta^{2}}{4} \theta^{2}\right)\left(p_{1}^{2}+p_{2}^{2}\right)+\left(\beta^{2}+\frac{\alpha^{2}}{4} \eta^{2}\right) \times$
$\times\left(x_{1}^{2}+x_{2}^{2}\right)-\left(\alpha^{2} \eta+\beta^{2} \theta\right)\left(x_{1} p_{2}-x_{2} p_{1}\right)$,

correspond to the Hamiltonian of a two-dimensional noncommutative harmonic oscillator. Therefore, in view of (99), the eigenvalues of $\mathbf{Q}^{2}$ read

$Q_{n_{1} n_{2} n_{3}}^{2}=$

$=\hbar\left(\sqrt{\left(2 \alpha^{2}+\frac{\theta^{2} \beta^{2}}{2}\right)\left(2 \beta^{2}+\frac{\eta^{2} \alpha^{2}}{2}\right)}+\eta \alpha^{2}+\theta \beta^{2}\right) n_{1}+$

$+\hbar\left(\sqrt{\left(2 \alpha^{2}+\frac{\theta^{2} \beta^{2}}{2}\right)\left(2 \beta^{2}+\frac{\eta^{2} \alpha^{2}}{2}\right)}-\eta \alpha^{2}-\theta \beta^{2}\right) n_{2}+$

$+\hbar \sqrt{\left(2 \alpha^{2}+\frac{\theta^{2} \beta^{2}}{2}\right)\left(2 \beta^{2}+\frac{\eta^{2} \alpha^{2}}{2}\right)}+$

$+2 \hbar \alpha \beta\left(n_{3}+\frac{1}{2}\right)$.

On the basis of this result, we can write the inequalities

$$
\begin{aligned}
& \left\langle\Delta \mathbf{Q}^{2}\right\rangle \geq \hbar \sqrt{\left(2 \alpha^{2}+\frac{\theta^{2} \beta^{2}}{2}\right)\left(2 \beta^{2}+\frac{\eta^{2} \alpha^{2}}{2}\right)}+ \\
& +\hbar \eta \alpha^{2}+\hbar \theta \beta^{2}+\hbar \alpha \beta, \\
& \Delta Q \geq\left\{\hbar\left(2 \alpha^{2}+\frac{\theta^{2} \beta^{2}}{2}\right)^{1 / 2}\left(2 \beta^{2}+\frac{\eta^{2} \alpha^{2}}{2}\right)^{1 / 2}+\right. \\
& \left.+\hbar \eta \alpha^{2}+\hbar \theta \beta^{2}+\hbar \alpha \beta\right\}^{1 / 2},
\end{aligned}
$$

with $\Delta Q=\sqrt{\left\langle\Delta \mathbf{Q}^{2}\right\rangle}$. So, the minimal length in the noncommutative phase space reads

$$
\begin{aligned}
& \Delta Q^{\min }=\left\{\hbar\left(2 \alpha^{2}+\frac{\theta^{2} \beta^{2}}{2}\right)^{1 / 2}\left(2 \beta^{2}+\frac{\eta^{2} \alpha^{2}}{2}\right)^{1 / 2}+\right. \\
& \left.+\hbar \eta \alpha^{2}+\hbar \theta \beta^{2}+\hbar \alpha \beta\right\}^{1 / 2} .
\end{aligned}
$$

Note that, by putting $\alpha=0$, we get $\Delta D^{\text {min }}=$ $=\Delta P^{\mathrm{min}}$, which is defined in (86). For $\beta=0$, we obtain $\Delta D^{\text {min }}=\Delta R^{\text {min }}$ presented by (58), as it has to be. Note that the noncommutativity of momenta (3) causes additional restrictions on the length comparing with that in the noncommutative space (space with the noncommutativity of coordinates, $\left.\eta_{i j}=0\right)$.

\section{Conclusions}

We have considered a noncommutative phase space realized with the help of the commutation relations (1)-(3). The lower bound on the length in the space has been studied. Particular cases of the definition of length in the coordinate and momentum spaces are examined. In each case, the restrictions on the length have been obtained, by analyzing the uncertainty relations for coordinates and momenta, on the basis of solutions of the eigenvalue problem for the squared length operator. Comparing with the ordinary space (space with commutative coordinates and commutative momenta), where the length is not restricted, the noncommutative space has a minimal length. It has been shown that noncommutativity (1)-(3) causes the existence of a lower bound of the length and also its anisotropy.

We have also studied the general case where the length is defined in the phase space. Based on the exact solution of the eigenvalue problem for the squared length operator, we have found an expression for the minimal length (111). It has been shown that, in the noncommutative phase space (space with the noncommutativities of coordinates and momenta), there are additional restrictions on the length comparing to that in the noncommutative space (space with the noncommutativity of coordinates).

This work was partly supported by the European Commission under the project STREVCOMS PIRSES-2013-612669 and the projects FF-63Hp (No.0117U007190), FF-30F (No.0116U001539) from the Ministry of Education and Science of Ukraine.

1. N. Seiberg, E. Witten. String theory and noncommutative geometry. J. High Energy Phys. 9909, 032 (1999).

2. S. Doplicher, K. Fredenhagen, J.E. Roberts. Spacetime quantization induced by classical gravity. Phys. Lett. B 331, 39 (1994).

3. J. Gamboa, M. Loewe, J.C. Rojas. Noncommutative quantum mechanics. Phys. Rev. D 64, 067901 (2001).

4. V.P. Nair, A.P. Polychronakos. Quantum mechanics on the noncommutative plane and sphere. Phys. Lett. B 505, 267 (2001).

5. K. Bolonek, P. Kosinski. On uncertainty relations in noncommutative quantum mechanics. Phys. Lett. B 547, 51 (2002).

6. C. Duval, P.A. Horvathy. Exotic Galilean symmetry in the non-commutative plane and the Hall effect. J. Phys. A 34, 10097 (2001).

ISSN 2071-0194. Ukr. J. Phys. 2018. Vol. 63, No. 2 
7. M. Chaichian, M.M. Sheikh-Jabbari, A. Tureanu. Hydrogen atom spectrum and the lamb shift in noncommutative QED. Phys. Rev. Lett. 86, 2716 (2001).

8. T.C. Adorno, M.C. Baldiotti, M. Chaichian, D.M. Gitman, A. Tureanu. Dirac equation in noncommutative space for hydrogen atom. Phys. Lett. B 682, 235 (2009).

9. J.M. Romero, J.D. Vergara. The Kepler problem and noncommutativity. Mod. Phys. Lett. A 18, 1673 (2003).

10. B. Mirza, M. Dehghani. Noncommutative geometry and classical orbits of particles in a central force potential. Commun. Theor. Phys. 42, 183 (2004).

11. O. Bertolami, P. Leal. Aspects of phase-space noncommutative quantum mechanics. Phys. Lett. B 750, 6 (2015).

12. O. Bertolami, J.G. Rosa, C.M.L. de Aragao, P. Castorina, D. Zappala. Scaling of variables and the relation between noncommutative parameters in noncommutative quantum mechanics. Mod. Phys. Lett. A 21, 795 (2006).

13. Kh.P. Gnatenko, V.M. Tkachuk. Effect of coordinate noncommutativity on the mass of a particle in a uniform field and the equivalence principle. Mod. Phys. Lett. A $\mathbf{3 1}$, 1650026 (2016).

14. Kh.P. Gnatenko. Estimating the upper bound of the parameter of noncommutativity on the basis of the equivalence principle. J. Phys. Stud. 17, 4001 (2013).

15. Kh.P. Gnatenko. Physical systems in a space with noncommutativity of coordinates. J. Phys.: Conf. Ser. 670, 012023 (2016).

16. H. Snyder. Quantized space-time. Phys. Rev. 71, 38 (1947).

17. A.E.F. Djemai, H. Smail. On quantum mechanics on noncommutative quantum phase space. Commun. Theor. Phys. 41, 837 (2004).

18. Li Kang, Chamoun Nidal. Hydrogen atom spectrum in noncommutative phase space. Chin. Phys. Lett. 23, 1122 (2006).

19. S.A. Alavi. Lamb shift and Stark effect in simultaneous space-space and momentum-momentum noncommutative quantum mechanics and $\theta$. Mod. Phys. Lett. A 22, 377 (2007).

20. O. Bertolami, R. Queiroz. Phase-space noncommutativity and the Dirac equation. Phys. Lett. A 375, 4116 (2011).

21. A. Smailagic, E. Spallucci. Isotropic representation of the noncommutative 2D harmonic oscillator. Phys. Rev. D 65 107701 (2002).

22. A. Smailagic, E. Spallucci. Noncommutative 3D harmonic oscillator. J. Phys. A 35, 363 (2002).

23. A. Hatzinikitas, I. Smyrnakis. The noncommutative harmonic oscillator in more than one dimension. J. Math. Phys. 43, 113 (2002).
24. Li Kang, Wang Jianhua, Chen Chiyi. Representation of noncommutative phase space. Mod. Phys. Lett. A 20, 2165 (2005).

25. C. Acatrinei. Path integral formulation of noncommutative quantum mechanics. J. of High Energy Phys. 9, 007 (2001).

26. P.R. Giri, P. Roy. The non-commutative oscillator, symmetry and the Landau problem. Eur. Phys. J. C 57, 835 (2008).

27. J. Ben Geloun, S. Gangopadhyay, F.G. Scholtz. Harmonic oscillator in a background magnetic field in noncommutative quantum phase-space. EPL 86, 51001 (2009).

28. O. Bertolami, J.G. Rosa, C.M.L. de Aragao, P. Castorina, D. Zappala. Noncommutative gravitational quantum well. Phys. Rev. D 72, 025010 (2005).

29. C. Bastos, O. Bertolami. Berry phase in the gravitational quantum well and the Seiberg-Witten map. Phys. Lett. A 372, 5556 (2008).

30. Kh.P. Gnatenko, V. M. Tkachuk. Weak equivalence principle in noncommutative phase space and the parameters of noncommutativity. Phys. Lett. A 381, 2463 (2017).

31. J.M. Romero, J.A. Santiago, J.D. Vergara. Note about the quantum of area in a noncommutative space. Phys. Rev. D 68, 067503 (2003).

32. A. Kijanka and P. Kosinski. Noncommutative isotropic harmonic oscillator. Phys. Rev. D 70, 127702 (2004).

33. Kh.P. Gnatenko, V.M. Tkachuk. Minimal length, area, and volume in a space with noncommutativity of coordinates. J. Phys. Stud. 20, 1001 (2016).

34. A.E.F. Djemai, H. Smail. On quantum mechanics on noncommutative quantum phase space. Commun. Theor. Phys. 41, 6 (2004).

Received 08.05.17

Х.П. Гнатенко, В.М. Ткачук

\section{ДОВЖИНА У НЕКОМУТАТИВНОМУ} ФАЗОВОМУ ПРОСТОРІ

$\mathrm{P}$ е $з$ ю м е

Вивчено обмеження на довжину у некомутативному фазовому просторі, зумовлені некомутативністю. Розглядаються співвідношення невизначеностей для координат та імпульсів та знаходиться нижня межа для довжини. Ми також розглядаємо задачу на знаходження власних значень оператора квадрата довжини та отримали вираз для мінімальної довжини у некомутативному фазовому просторі. 